\title{
Endoscopic treatment of Zenker's diverticulum with Ligasure: simple, safe and effective $\square$
}

\section{다 (용}

\author{
Authors \\ Pilar Diez Redondo, Henar Núñez Rodríguez, Marina de Benito Sanz, Raúl Torres Yuste, Manuel Pérez-Miranda
}

Institution

Servicio de Gastroenterología. Hospital Universitario del Río Hortega, Valladolid, España

submitted 9.7.2018

accepted after revisión 15.10.2018

Bibliography

DOI https://doi.org/10.1055/a-0809-4875 |

Endoscopy International Open 2019; 07: E203-E208

(c) Georg Thieme Verlag KG Stuttgart · New York ISSN 2364-3722

Corresponding author

Pilar Diez Redondo, Hospital Universitario Rio Hortega -

Digestive Diseases, Calle Dulzaina 2, Valladolid 47012,

Spain

Fax: +983420400

diezmp@hotmail.com

\section{ABSTRACT}

Background and study aims Zenker's diverticulum may cause disabling symptoms, especially in the elderly. Treatment has changed in recent decades from open surgery to management with flexible endoscopy, resulting in lower morbidity and mortality. The goal of this study was to pres- ent the largest series, with the longest follow-up, of patients with Zenker's diverticulum receiving outpatient treatment with flexible endoscopy using a diverticuloscope and Ligasure (Covidien, Minneapolis, Minnesota, United States), a device that allows tissue sealing and coagulation of vessels before cutting the septum between the diverticulum and esophagus.

Patients and methods We performed 79 diverticulotomies in 69 patients (65.2\% male, mean age 73.4 years). The mean diverticulum size was $2.8 \mathrm{~cm}$. In three cases with a diverticulum $\leq 1.5 \mathrm{~cm}$, the diverticuloscope could not be placed.

Results The technical success was $95.83 \%$ and the clinical success $96.7 \%: 84 \%$ of the 56 patients followed for a mean of 34.6 months (24-64 months) had no dysphagia. The recurrence rate was $10.4 \%$, with a good response to a second diverticulotomy at 12 months (IQR: 11.5-17) in most cases. The most severe complications were two microperforations, resolved with conservative treatment, and one case of delayed bleeding endoscopically-controlled with a clip.

Conclusions Diverticulotomy of the esophageal-diverticular septum with Ligasure is an outpatient endoscopic technique that is simple, effective in the long term and very safe for the treatment of patients with Zenker's diverticulum. In symptomatic recurrences, a second procedure was equally safe and effective in most patients.

\section{Introduction}

Zenker's diverticulum (ZD) is a saccular formation that protrudes into the posterior wall of the pharyngo-esophageal junction in an anatomically weak area between the inferior pharyngeal constrictor and cricopharyngeus muscles.

Conventional treatment of symptomatic ZD by open surgery (OS) to extirpate the ZD by cricopharyngeal myotomy has an overall postoperative morbidity rate of $11 \%$ [1], including mediastinitis (3\%), vocal cord paralysis, and pharyngocutaneous fistula, among others, and the mortality rate is up to $3 \%$ [2]. Postsurgical recurrence rates are between $3 \%$ and $19 \%$, rising to $15 \%$ to $35 \%$ when there is no cricopharyngeal myotomy [3].
In the mid-1960 s, rigid endoscopy (RE), described and abandoned in previous decades due to its high mortality, was reintroduced using rigid diverticuloscopes. The greatest limitations of RE are the difficult maneuverability in patients with a small ZD and the need for maximum cervical hyperextension, with a large number of failed cases [4]. The recurrence rate is $10 \%$ to $12 \%$ and the complication rate $7 \%$ [5].

Since the first diverticulotomies with flexible endoscopy (FE) of the esophageal-diverticular septum (EDS) were described in 1995 [6], various endoscopic techniques have been developed with good results and less morbidity and mortality than surgery [7]. Different scalpels, monopolar coagulation forceps, laser and argon plasma have been used, together with use of transparent caps and flexible diverticuloscopes. In recent years, Li- 


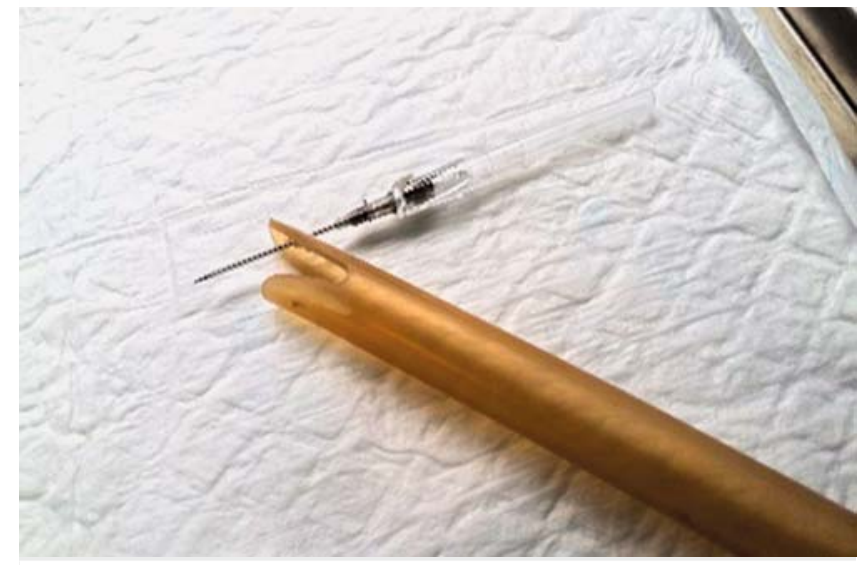

- Fig. 1 The external end of the guidewire inserted through a hole made through an $18 \mathrm{G}$ catheter at the end of the long blade of the plastic diverticuloscope (Cook Medical, Limerick, Ireland).

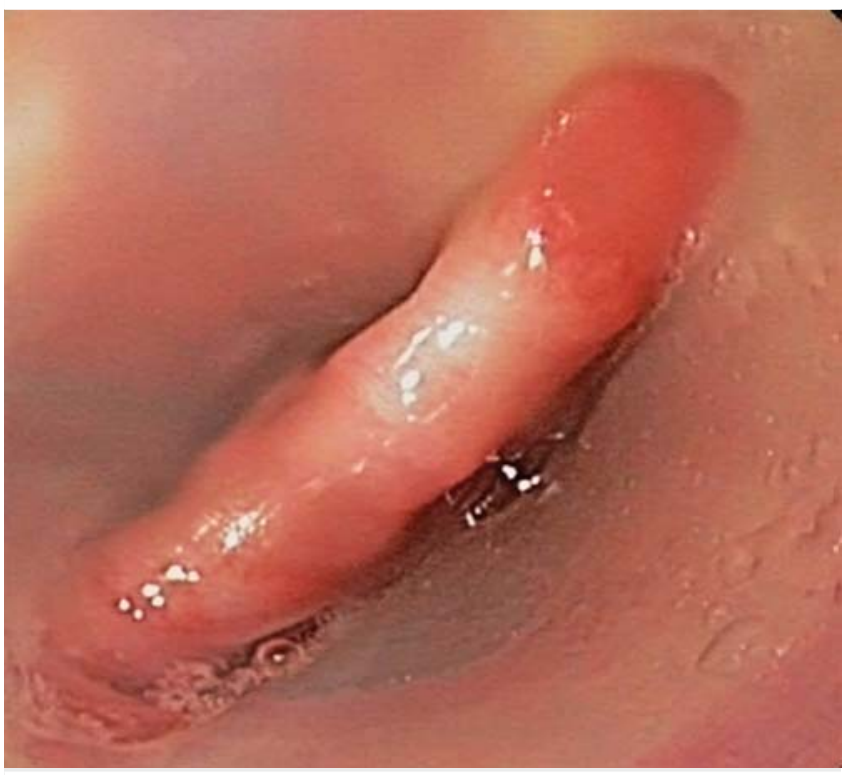

- Fig. 2 The esophageal-diverticular septum was isolated between the two blades after gentle advance of the diverticuloscope until the long blade fit into the esophageal lumen and the short blade into the diverticulum.

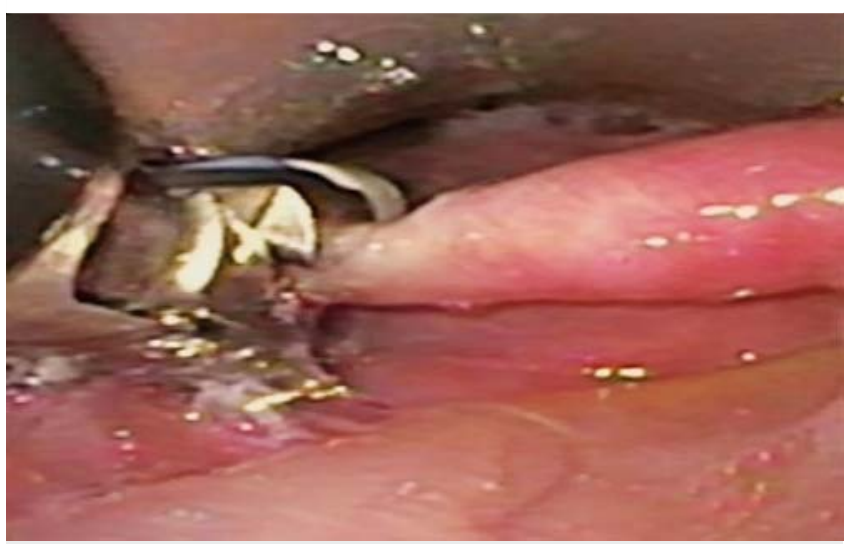

- Fig. 3 When the Ligasure reached the middle zone of the EDS, the blades were opened and closed on the tissue. gasure (LS1500, Covidien, Medtronic, Minneapolis, Minnesota, United States), a system imported from laparoscopic surgery, has shown very good results in small series [8-10]. We present the largest series of patients with ZD treated by endoscopic diverticulotomy with Ligasure (EDVL) with a long follow-up.

\section{Patients and methods}

We conducted 79 EDVLs between June 2010 and March 2018 in 69 patients with ZD in the Digestive Service, Río Hortega University Hospital, a tertiary health center. We prospectively collected sociodemographic, surgical, complications and followup data.

EDVLs were performed by three endoscopists on an outpatient basis under deep sedation with propofol controlled by the endoscopist, prophylactic antibiotic therapy and insufflation with $\mathrm{CO}_{2}$.

Ligasure is a bipolar device in the shape of a rotatable clamp that releases high current/low-voltage energy to the tissue trapped between its blades, producing tissue dissection and sealing of blood vessels $\leq 7 \mathrm{~mm}$, which ensures hemostasis prior to cutting the tissue.

The ZD was identified by gastroscopy, and a guidewire (Jagwire HPG, Boston Scientific, Natick, Massachusetts, United States) was placed in the gastric cavity before the gastroscope was retired. The external end of the guidewire was inserted through a hole made through an 18G catheter at the end of the long blade of the plastic diverticuloscope (PD) (Cook Medical, Limerick, Ireland) ( $\triangleright$ Fig. 1). Subsequently, the PD was introduced into the oral cavity and a gastroscope (GIFQ 180, Olympus Optical Co. Tokyo, Japan) was placed inside to provide endoscopic vision. The PD was advanced until the long blade fit into the esophageal lumen and the short blade into the diverticulum, allowing the EDS to be isolated between the two blades ( $\triangleright$ Fig. 2), and then the gastroscope was removed and the Ligasure $(5 \mathrm{~mm} \times 35 \mathrm{~mm})$, connected to the generator, inserted within the $\mathrm{PD}$ in parallel with a $4.5-\mathrm{mm}$ gastroscope (GIFXP160N Olympus) to guide its advance and permit the ZD to be measured.

When the Ligasure reached the middle zone of the EDS, the blades were opened and closed on the tissue ( $\triangleright$ Fig. 3 ), the clotting-tissue sealing function was activated and the cut was made ( $\triangleright$ Fig.4). This twin maneuver was repeated as often as necessary, depending on the size of the septum, to leave a residual wall $<5 \mathrm{~mm}$ to avoid perforations. A clip (Resolution Clip, Boston Scientific, Natick Massachusetts, United States) could be inserted preventively at the discretion of the endoscopist ( $\triangleright$ Fig. 5, $\triangleright$ Video 1). Post-procedure, patients were monitored and discharged to home in about 60 minutes, with a report on the intervention and written recommendations including initiating liquid intake at 8 hours post-intervention, soft foods in the following 24 hours and a progressively normal intake during the first week, and instructions to visit the emergency room if necessary. Patients received a telephone call from the endoscopy team at 24 hours, 1 week, 1 month and 6 months and, where possible, clinical or telephone follow-up was maintained for up to 4 years. An objective dysphagia scale could not be 
used because a large part of the follow-up was by phone and many patients were elderly and did not understand the numerical scale we wished to employ.

The statistical analysis was made using the Stata program (StataCorp 2013, Stata Statistical Software Release 13, StataCorp LP, College Station, Texas, United States). Categorical variables were described as percentages. Continuous variables were presented as mean and standard deviation if the distribution was normal and as median, interquartile range (IQR) and/ or range when the distribution was non-normal.

\section{Results}

Between June 2010 and March 2018, 72 patients with symptomatic ZD were referred to our Endoscopy Unit for EDVL. There were 45 men and 24 women, with a mean age of 73.4 years (SD: 11.1). One patient had previously undergone surgery for ZD. Patients reported symptoms for a mean of 1.5 years (IRQ: $1-2$ ) before referral for treatment. The most frequent symptom was dysphagia in $73.9 \%$ of the cases, which was very severe, preventing fluid intake, in 11 cases, severe for solid foods in 40 and mild or intermittent in 18 . Forty-five patients had food regurgitation from the diverticulum and there were 19 episodes of choking, 16 halitosis, 9 nocturnal episodes of cough or regurgitation, 8 cases with weight loss, and aspiration pneumonia in 10 cases ( $>$ Table $\mathbf{1})$.

Technical success was defined as EDS section using EDVL. Clinical success was defined as patient-reported disappearance of symptoms or significant symptomatic improvement 1 week after EDVL. Recurrence was defined as the reappearance of daily or weekly symptoms in asymptomatic patients or occasional symptomatology after the intervention.

In three $Z D \leq 1.5 \mathrm{~cm}$, EDVL was not possible because the PD could not be placed. There was no failure due to inability to manipulate the Ligasure. EDVL was performed in 69/72 patients, with a technical success of $95.83 \%$. Mean ZD size was $2.8 \mathrm{~cm}$ (SD $1.1 \mathrm{~cm}$ ) and median number of cuts was two (IQR: $1-2$ ), depending on the length of the EDS. In 49 cases (71\%) a clip was placed before retiring the overtube. The mean time of EDVL, from introduction of the gastroscope to extraction of the PD from the oral cavity, was 22 minutes (11-48).

Ten patients continued with or presented dysphagia after EDVL and underwent a second EDVL. Therefore, duringn the study period, we performed 79 EDVLs in 69 patients.

Immediate complications were two case $(2.5 \%)$ of mild, selflimited intraprocedural bleeding after cutting the septum.

Most early complications were mild: odynophagia (21.5\%) and cervical pain ( $5 \%$ ) that resolved in $<72$ hours with first-level analgesia. However, two males $(2.5 \%)$ presented iatrogenic esophageal microperforation in the first hours after treatment, requiring hospital admission for 8 and 12 days, respectively. These patients reported thoracic or interscapular pain and low-grade fever with leukocytosis, and small peridiverticular air bubbles were evident on computed tomography, despite placement of a clip in both cases. Neither required surgical or endoscopic procedures, and evolved well with conservative treatment that included fasting and antibiotic therapy. One pa-

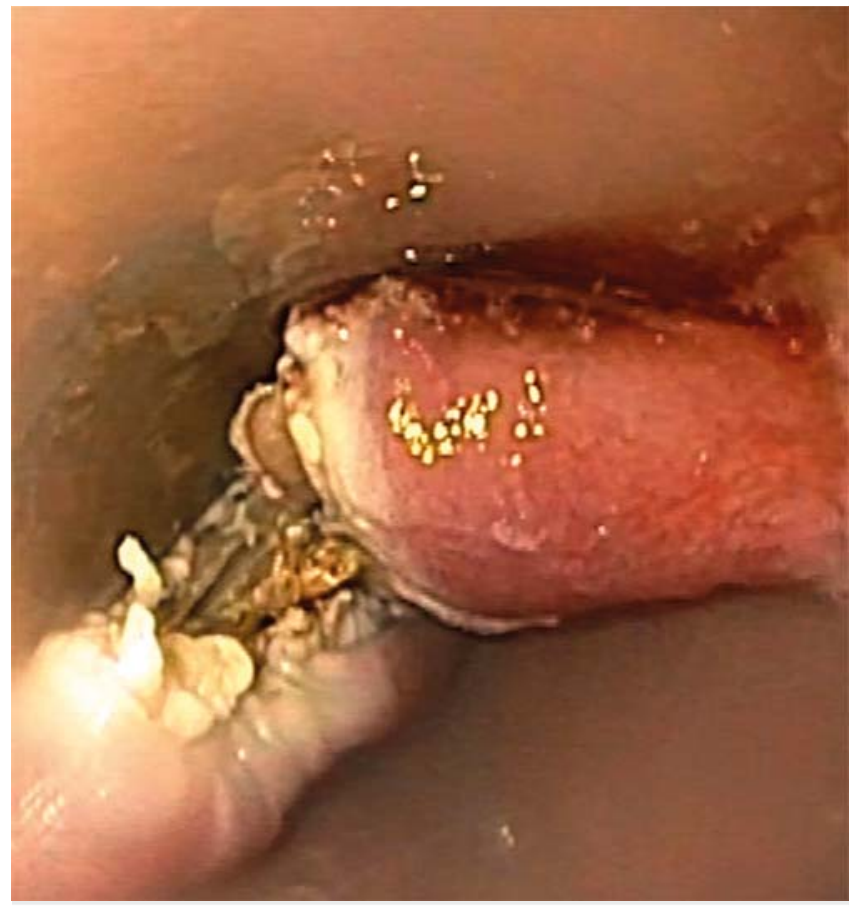

Fig. 4 Appearance of the septum after cutting with Ligasure.

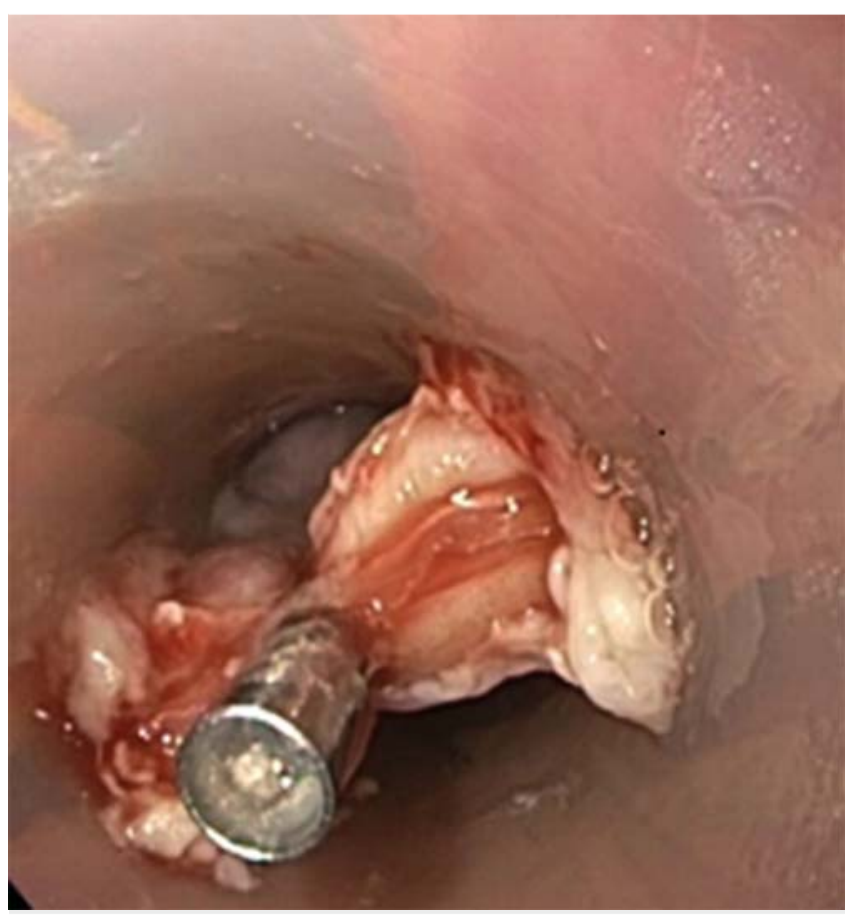

Fig. 5 After cutting, a clip (Resolution Clip, Boston Scientific, Natick Massachusetts, United StatesA) can be inserted, depending on the preference of the endoscopist, to avoid perforations.

tient, with a 2-cm ZD, who received a single cut, had ankylosing spondylitis with significant deformity and cervical-thoracic rigidity that made maneuvers difficult during the intervention. The other patient, with a $3.5-\mathrm{cm}$ ZD, was treated with two cuts, without abnormalities being detected during the intervention. 


\begin{tabular}{|l|c|}
\hline Table 1 Patient data n (\%). & $\mathrm{n}=69$ \\
\hline Total number of patients & $24(34.8 \%)$ \\
\hline Female & $45(65.2 \%)$ \\
\hline Male & $73.4(46-93)$ \\
\hline Age, years, mean (range) & $1.5(1-2)$ \\
\hline Symptom duration before treatment (years) & \\
\hline Symptoms & \\
\hline Dysphagia: & $11(15.9 \%)$ \\
\hline - Very severe (fluids) & $40(58 \%)$ \\
\hline Severe (solid foods) & $18(26.1 \%)$ \\
\hline Regurgitation & $45(65.2 \%)$ \\
Choking & $19(27.5 \%)$ \\
\hline Halitosis & $16(23.2 \%)$ \\
\hline Nocturnal cough or regurgitation & $9(13 \%)$ \\
\hline Weight loss & $8(11.6 \%)$ \\
\hline Aspiration pneumonia & $10(14.5 \%)$ \\
\hline
\end{tabular}

The only late complication (1.3\%) was a case of iatrogenic digestive hemorrhage, 10 days after EDVL, without hemodynamic repercussions, treated endoscopically with a clip ( $\triangleright$ Table 2).

The 69 patients or their relatives were contacted by telephone the day after EDVL and subsequently, with a progressive loss of cases: 61 (85.5\%) at 1 week, 51 (73.9\%) at 1 month, 36 $(52.2 \%)$ at 6 months and $56(81.2 \%)$ at 34.6 months on average (24-64 months).

At 8 hours, 53/69 patients ( $77.8 \%$ ) had started a liquid diet, and the 61 located at 1 week, except for the two with microperforation, tolerated a solid or semi-solid diet, indicating a tolerance to more solid food than before the intervention, with disappearance or improvement of other symptoms such as regurgitation or cough. Therefore, the clinical success rate was $96.7 \%(59 / 61)$.

One month after EDVL, 44/51 (86.3\%) patients had no dysphagia and $7 / 51(13.7 \%)$ reported difficulty in eating some solids (especially fibrous meats or bread).

At 6 months, 33 of $36(91.6 \%)$ reported remaining asymptomatic and 3 of 36 (8.3\%) reported dysphagia for solid food, with one patient with little initial improvement and very fre-

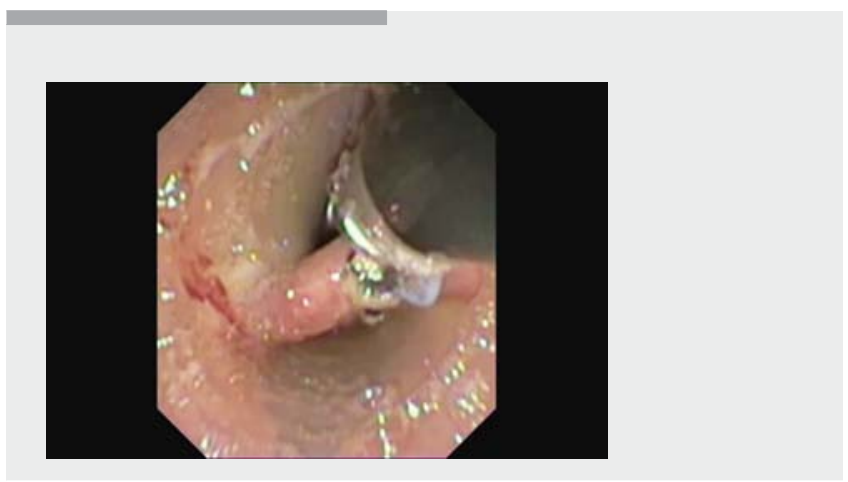

Video 1 Procedure of Zenker diverticulotomy.

\begin{tabular}{|l|c|}
\hline \begin{tabular}{l} 
Table 2 Technical results and complications. \\
\hline Successful septotomy, number of cases
\end{tabular} & $69 / 72(95.83 \%)$ \\
\hline Procedure time, minutes, mean (range) & $22(11-48)$ \\
\hline Septum size in cm, mean (range) & $2.8(1-5)$ \\
\hline Number of cuts, mean (range) & $2(1-4)$ \\
\hline Used clips (number of cases ) & $49(71 \%)$ \\
\hline \begin{tabular}{l} 
Immediate complications (intraprocedure) \\
\hline - Self-limited bleeding
\end{tabular} & $2(2.5 \%)$ \\
\hline \begin{tabular}{l} 
Early complications (24-48 h after procedure) \\
\hline - Odynophagia
\end{tabular} & $21(30 \%)$ \\
\hline - Cervical pain & $15(21.5 \%)$ \\
\hline Esophageal microperforation & $4(5 \%)$ \\
\hline $\begin{array}{l}\text { Late complications (> } 72 \mathrm{~h} \text { after procedure) } \\
\text { Hemorrhage }\end{array}$ & $1(2.5 \%)$ \\
\hline
\end{tabular}

quent dysphagia. This patient was considered as "insufficient treatment" and underwent a second EDVL with a very good evolution.

Of the 56 patients (81.2\%) followed for a mean of 34.6 months, 47 (84\%) had no dysphagia (including the patient with a second early EDVL), four had dysphagia only for fibrous foods, three dysphagia for semisolids and regurgitation, and two severe dysphagia.

The nine patients who reported recurrence of dysphagia, after initially having been asymptomatic or with significant symptom improvement, underwent a second EDVL after a median of 12 months (IQR: 11.5 -17, range 10-64) after the first procedure, with clinical success in seven cases. No improvement in dysphagia was achieved in two elderly women with senile dementia who, although presenting initial improvement, had progressive worsening which did not respond to enlargement of the small residual EDS cut. These patients presented severely diminished esophageal motility with a "corkscrew appearance" and food remains along the esophagus. This was compatible with an age-related reduction in esophageal motility but, due to the advanced age and inability to collaborate, no esophageal manometry study was made. The first patient received placement of a percutaneous endoscopic gastrostomy, which was rejected by the second patient's family.

Without considering the patient with a bad response to the first treatment and the two elderly patients with progressive early worsening and no improvement after a second treatment, primary success with recurrence, i.e. asymptomatic cases or clear improvement after the first EDVL with reappearance of symptoms months or years later without other reasons for dysphagia, occurred in 7 of 69 patients (10.4\%).

In patients with recurrence, the mean size of the ZD before the first intervention was $2.8 \mathrm{~cm}$ (range $1-5$, SD $1.1 \mathrm{~cm}$ ) and the median number of cuts was 2 (IQR: $1-2$ ), with a clip placed in $60 \%$ after the EDS section. The mean size of the ZD after the first EDVL in the seven patients where information was available was 1.5 (range $0.9-2.5$ ) 
One year after the second EDVL, three patients had died, two were not contacted, three were asymptomatic, one reported occasional dysphagia for some hard foods and one patient still had severe dysphagia.

\section{Discussion}

Our results in 69 outpatients with ZD with FE show that Ligasure is a simple and rapid technique with few complications and lasting efficiency.

We used a PD that permits a better vision and fixation of the EDS with more precise control of the length of the cut and which was also very useful in directing the placement of a clip and reducing risk of aspiration during the procedure. In three patients with ZD $<1.5 \mathrm{~cm}$ we were unable to fit the EDS between the two valves of the PD because the short blade, which measures $2.5 \mathrm{~cm}$, was longer than the diverticulum. It might be useful to design PDs with shorter blades or use a cap in a very small DZ, which might be the only limitation of this method. However, a study comparing a cap with PD found a similar technical success rate but significantly greater clinical success in patients in whom PD was used, with a greater number of adverse events (AEs) and a longer intervention time with use of the cap [11]. Once the PD was placed, there were no specific difficulties in introducing the Ligasure or in sectioning the EDS, regardless of its thickness, as has been reported with use of some staplers [12]. The procedure required a mean of 22 minutes, among the shortest reported times for EF $[12,13]$.

Our series had a technical success rate of $95.8 \%$, with $96.7 \%$ clinical success and $84 \%$ asymptomatic patients at 34.6 months (24-64 months). The recurrence rate was $10.4 \%$ at 20.3 months (10-64 months) with a good response to a second EDVL at 12 months (IQR: 11.5-17). A second EDVL achieved disappearance or frank reduction in symptoms in a male with poor initial improvement after EDVL and seven patients with symptomatic recurrence. Nevertheless, two elderly women with senile dementia and age-related severely decreased esophageal peristalsis did not improve after a second procedure on a small residual ZD. This shows that a second, rescue $E D V L$ is very useful in patients with incomplete treatment or recurrence, provided they are well selected, which probably excludes those with severe comorbidities and other disorders of swallowing or esophageal motility, as recommended by other authors [14]. In our series, there was only one postsurgical recurrence, which was managed successfully without complications. We believe that EDVL is suitable in this context as are other endoscopic techniques [2,15]. Unfortunately, we were not able to measure improvement in symptoms using an objective scale and had to rely on subjective assessment by the patients.

A review [1] of FE techniques, not including Ligasure found a global technical success rate of $99.4 \%$, a clinical success rate of $87.9 \%$ (after 1 to 3 treatment sessions), a failure rate due to persistence of symptoms of $10 \%$ and a recurrence rate of $13.5 \%$, with a mean/median follow-up ranging from 7 to 43 months.
There are few reports on Ligasure for treatment of ZD, with the first dating from $2014[8,16]$. Two small series $[9,10](n=5$ and 8 ) reported no recurrences after a mean follow-up of about 21 months, including a partial clinical improvement efficiently rescued with a second EDVL at 3 months. The procedures were performed, as in our series, under deep sedation with propofol, but patients were hospitalized for $\geq 24$ hours while our patients were discharged after the procedure.

A study [16] of 15 patients with ZD treated with Ligasure with RE under general anesthesia reported $93 \%$ of asymptomatic patients at 2 to 8 weeks post-procedure with $13.3 \%$ recurrences after a mean follow-up of 9 months and found that Ligasure was much easier to manipulate than surgical staplers. In 2017, the same group reported a 22-month follow-up of 21 patients with $57 \%$ free from dysphagia and $38 \%$ asymptomatic [17].

Symptomatic recurrence may be due to incomplete section of the cricopharyngeus muscle or subsequent scarring. Risk factors associated with recurrence are a $Z D \geq 5 \mathrm{~cm}$, an EDS cut $\leq 25 \mathrm{~mm}$ and a post-treatment ZD $\geq 1 \mathrm{~cm}$ [18]. In our cases with recurrence we found no differences in ZD size or number of cuts made compared with patients without recurrence, although the mean size of the septum before the second treatment in the seven patients where this information was recorded was $1.5 \mathrm{~cm}$ (range $0.9-2.5$ ). As we did not make endoscopic controls, we cannot compare this measurement in asymptomatic patients. Leaving the smallest septum without causing a perforation seems to be crucial to obtaining good symptomatic control and reducing recurrence. Variants of the usual section technique with greater resection of the ESD [19-21] and its resection by submucosal tunneling [22] have recently been described, but prospective data are needed to determine the reproducibility and long-term effectiveness of this technique.

The most frequent complications secondary to FE treatment for ZD [15] are hemorrhage (6.6\%) either intraprocedural or post-intervention, and perforation/emphysema (5.3\%) requiring surgery in $0.9 \%$, with no mortality being described.

Our intraprocedural bleeding rate was $2.5 \%$, much lower than that described with other endoscopic devices. An intraprocedural bleeding rate with different types of needle-knife of $33 \%$ has been described, requiring use of argon plasma or monopolar electrocoagulation for resolution and sometimes preventing successful therapy due to lack of visibility [14]. Our few episodes of mild, self-limited bleeding may probably be considered intraprocedural events without technical or clinical repercussions rather than $\mathrm{AEs}$ and this is one of the main strengths of use of Ligasure, in line with some stapler devices [12] and clearly safer, with respect to bleeding, than other scalpels. We had one case of slight delayed bleeding 10 days postintervention, which was resolved endoscopically.

Our perforation rate of $2.53 \%$ is lower than the $5.3 \%$ described with other FE techniques without tissue sealing [15], while in some series $[23,24]$ with the needle-knife it is higher than $20 \%$, occasionally requiring surgical treatment or the insertion of endoscopic stents. Our two microperforations were resolved conservatively. Placement of clips, initially described by Tang [25], which was used in $71 \%$ of our cases, may help pre- 
vent perforations $[14,18]$ and therefore is routinely recommended by some authors, although cases of microperforation have been described despite sealing with clips [26], as occurred in our two cases. Other authors [27] prefer not to use clips to avoid scar formation but it is unclear whether this is related to worse clinical outcomes.

\section{Conclusion}

In conclusion, our results reinforce the few data available on treatment of ZD with FE and Ligasure, demonstrating that it is a simple, effective, and very safe ambulatory technique that also permits resolution of the majority of recurrences in wellselected patients. Although consensus on the best endoscopic option for treatment of ZD in each patient would be ideal, after direct comparison between the different devices, we can currently recommend Ligasure as one of the simplest and safest.

\section{Competing interests}

\section{None}

\section{References}

[1] Yuan Y, Zao YF, Hu Y et al. Surgical treatment of Zenker's diverticulum. Dig Surg 2013; 30: 207

[2] Antonello A, Ishaq S, Zanatta L et al. The role of flexible endotherapy for the treatment of recurrent Zenker's diverticula after surgery and endoscopic stapling. Surg Endosc 2016; 30: 2351-2357

[3] Gutschow CA, Hamoir M, Rombaux P et al. Management of pharyngoesphageal (Zenker's) diverticulum: which technique? Ann Thorac Surg 2002; 74: 1677

[4] Bloom JD, Bleier BS, Mirza N et al. Factors predicting endoscopic exposure of Zenker's diverticulum. Ann Otol Rhinol Laryngol 2010; 119: 736

[5] Chang CY, Payyapilli RJ, Scher RL. Endoscopic staple diverticulostomy for Zenker's diverticulum. Review of literature and experience in 159 consecutive cases. Laryngoscope 2003; 113: 957

[6] Ishioka S, Sakai P, Maluf Filho F et al. Endoscopic incision of Zenker's diverticula. Endoscopy 1995; 27: $433-437$

[7] Ishaq S, Sultan H, Siau K et al. New and emerging techniques for endoscopic treatment of- Zenker's diverticulum: state of-the-art review. Digestive Endoscopy 2018: Feb 9

[8] González N, Viola M, Costa X et al. Endoscopic treatment of Zenker's diverticulum by Ligasure scalpel. Endoscopy 2014; 46: E229-E230

[9] Noguera Aguilar ], Dolz Abadía C, Vilella A et al. Transoral endoluminal approach to Zenker's diverticulum using Ligasure. Early clinical experience. Rev Esp Enferm Dig 2014; 106: 137 - 141
[10] Moreira da Silva BA, Germade A, Pérez Cítores L et al. Endoscopic diverticulotomy using Ligasure. Gastroenterol Hepatol 2017; 40: $80-84$

[11] Costamagna G, lacopini F, Tringali A et al. Flexible endoscopic Zenker's diverticulotomy: cap-assisted technique vs. diverticuloscopeassisted technique. Endoscopy 2007; 39: 146-152

[12] Wilsen J, Baumbach R, Stüker D et al. New flexible endoscopic controlled stapler technique for the treatment of Zenker's diverticulum: a case series. World J Gastroenterol 2017; 23: 3084-3091

[13] Jain D, Sharma A, Shah M et al. efficacy and safety of flexible endoscopic management of Zenker's diverticulum. J Clin Gastroenterol 2018; 52: 369-385

[14] De la Morena Madrigal E, Pérez-Arellano E, Rodríguez-García I. Flexible endoscopic treatment of Zenker's diverticulum: thirteen years' experience in Spain. Rev Esp Enferm Dig 2016; 108: 297 - 303

[15] Ishaq S, Hassan C, Antonello A et al. Flexible endoscopic treatment for Zenker's diverticulum: a systematic review and meta-analysis. Gastrointest Endosc 2016; 83: 1076 - 1089:e5

[16] Nielsen HU, Trolle W, Rubek N et al. New technique using Ligasure for endoscopic mucomyotomy of Zenker's diverticulum: Diverticulotomy made easier. Laryngoscope 2014; 124: 2039-2042

[17] Andersen MF, Trolle W, Anthonsen K et al. Long-term results using Ligasure $5 \mathrm{~mm}$ instrument for treatment of Zenker's diverticulum. Eur Arch Otorhinolaryngol 2017; 274: 1939-1944

[18] Costamagna G, lacopini F, Bizzotto A et al. Prognostic variables for the clinical success of flexible endoscopic septotomy of Zenker's diverticulum. Gastrointest Endosc 2016; 84: 765 - 773

[19] Battaglia G, Antonello A, Realdon S et al. Flexible endoscopic treatment for Zenker's diverticulum with the SB knife. Preliminary results from a single center experience. Dig Endosc 2015; 27: 728 - 733

[20] Gölder SK, Brueckner J, Ebigbo A et al. Double incision and snare resection in syntomatic Zenker's diverticulum: a modification of the stag beetle knife technique. Endoscopy 2018; 50: 137-141

[21] Brahmbhatt B, Bartel M], Bhurwal A et al. Novel technique for flexible endoscopic repair of Zenker's diverticulum. videoGIE 2016; 1: 51 - 52

[22] Brieau B, Leblanc S, Bordacahar B et al. Submucosal tunneling endoscopic septum division for Zenker's diverticulum: A reproducible procedure for endoscopist who perform peroral endoscopic myotomy. Endoscopy 2017; 49: 613-614

[23] Vogelsang A, Preiss C, Neuhaus $\mathrm{H}$ et al. Endotherapy of Zenker's diverticulum using the needle-knife technique: long-term follow up. Endoscopy 2007; 39: 131-136

[24] Case DJ, Baron TH Eds. Flexible endoscopic management of Zenker's diverticulum: The Mayo Clinic experience. Mayo Clinic Proc 2010; 85: $719-722$

[25] Tang SJ, Jazrawi SF, Chen E et al. Flexible endoscopic clip-assisted Zenker's diverticulotomy: the first case series. Laringoscope 2008; 118: $1199-1205$

[26] Aiolfi A, Scolari F, Saino G et al. Current status of minimally invasive endoscopic management for Zenker's diverticulum. World ] Gastrointest Endosc 2015; 7: 87-93

[27] Gölder SK. Differences in endoscopic techniques for symptomatic Zenker's diverticulum. Endoscopy 2018; 50: 183-184 